\title{
Identificação de itens alimentares constituintes da dieta dos peixes-boi marinhos (Trichechus manatus) na região Nordeste do Brasil
}

\author{
João Carlos Gomes Borges ${ }^{1 *}$ \\ Patrícia Guimarães Araújo \\ Daiane Garcia Anzolin ${ }^{3}$ \\ George Emmanuel Cavalcanti de Miranda ${ }^{4}$ \\ Programa de Pós Graduação em Ciência Veterinária, UFRPE \\ Fundação Mamíferos Aquáticos, FMA \\ Rua Manoel Andrade, 2460, Coroa do Meio, CEP 49035-530, Aracaju - SE, Brasil \\ ${ }^{2}$ Programa de Pós-Graduação em Oceanografia, UFPE \\ ${ }^{3}$ Universidade do Vale do Itajaí, UNIVALI \\ ${ }^{4}$ Universidade Federal da Paraíba. Programa de Pós-Graduação em Oceanografia - UFPE \\ *Autor para correspondência \\ jcgborges@hotmail.com
}

\section{Resumo}

Os peixes-boi marinhos são considerados herbívoros oportunistas, consumindo uma grande variedade de itens alimentares. Objetivou-se com este trabalho identificar os itens alimentares constituintes da dieta dos peixes-boi marinhos em vida livre, na região Nordeste do Brasil. Para tanto foram coletadas amostras de conteúdo estomacal, provenientes de seis carcaças de peixes-boi marinhos, além de amostras fecais de 11 animais nativos e cinco espécimes reintroduzidos. O material foi identificado ao nível de gênero e/ou espécie, através de aspectos morfo-anatômicos, sendo identificadas 21 espécies entre macroalgas, fanerógamas e cnidários. Através destas análises, foi possível observar que os peixes-boi marinhos alimentaram-se de uma grande diversidade de plantas aquáticas, com predominância das algas vermelhas.

Unitermos: mamíferos aquáticos, sirênios, macroalgas, fanerógamas, alimentação

\section{Abstract}

Identification of alimentary components of Antillean manatee diet in the Northeast of Brazil. The manatees are considered to be opportunist herbivors, consuming a great amount of alimentary items. The aim of the present study was to identify the alimentary components of the diet of Antillean manatees in free life in the northeastern region of Brazil. Samples of stomach contents from six Antillean manatee carcasses were collected, in addition to fecal samples from 11 native manatees and five that had been released. The material was identified at the genus and/or species level, based on its morpho-anatomic aspects, and 21 species of seaweeds, phanerogams and cnidarians were presented. Through these analyses it was possible to observe that Antillean manatees fed on a great variety of aquatic plants, with the predominance of red seaweeds.

Key words: aquatic mammals, sirenians, seaweed, phanerogams, alimentary 


\section{Introdução}

Considerado o mamífero aquático mais ameaçado de extinção no Brasil, o peixe-boi marinho (Trichechus manatus) apresenta a sua população distribuída de forma descontínua nas regiões Norte e Nordeste (Lima, 1997; Luna, 2001). Em estudos realizados com peixes-boi marinhos na Flórida, os animais passaram até oito horas por dia alimentando-se, sendo considerados consumidores herbívoros e oportunistas (Hartman, 1979). Nestes locais de alimentação, intercalam-se segmentos de mar aberto e praias protegidas por recifes, que aliadas a águas transparentes, temperaturas e salinidades elevadas, permitem o desenvolvimento de uma rica e diversificada flora marinha, em especial de algas (Matos et al., 1998), que juntamente com as fanerógamas, abrigam um meiofauna abundante (Hicks, 1985).

Estudos prévios envolvendo populações de peixesboi marinhos e dugongos (Dugong dugon), foram feitos através de observação direta dos animais em atividades de alimentação (Hartman, 1979; Paludo e Langguth, 2002; Sheppard et al., 2007), sendo constatado nestas ocasiões, a presença de fanerógamas submersas, tais como, Thalasia testudinum, Syringodium filiforme, Halodule wrightii e Halophila engelmani. Além deste método de estudo, Burn (1986), através da análise do conteúdo do trato digestivo de peixes-boi marinhos na Flórida, identificou diferentes espécies de algas, arbustros e invertebrados, enquanto que Ames et al. (1996) e posteriormente Reich e Worthy (2006) utilizaram a técnica de isótopo de carbono para a determinação dos itens alimentares.

Segundo Paludo (1997), o método mais seguro para identificar a dieta alimentar de peixes-boi marinhos, seria através da análise dos itens ingeridos presentes no trato digestivo. A avaliação do conteúdo alimentar foi inicialmente desenvolvida por Baumgartner e Martin (1939) para determinar os hábitos alimentares de esquilos, sendo posteriormente aplicada em pesquisas com os sirênios, conforme observado em estudos com dugongos (Heinsohn e Birch, 1972; Wake, 1975; Marsh et al., 1982), peixes-boi amazônicos, Trichechus inunguis (Colares e Colares, 2002) e peixes-boi marinhos na Flórida (Burn, 1986; Ledder, 1986).
A obtenção de informações referentes à dieta alimentar dos peixes-bois marinhos de vida livre, além de gerar novos esclarecimentos sobre a bioecologia da espécie, favorece no processo de conservação destes animais, propiciando subsídios para a definição do manejo alimentar dos espécimes mantidos em cativeiro.

Este trabalho objetivou identificar os itens alimentares constituintes da dieta dos peixes-bois marinhos em vida livre na região Nordeste do Brasil.

\section{Material e Métodos}

As atividades foram realizadas de fevereiro de 2003 a março de 2005, onde coletou-se amostras de conteúdo estomacal, a partir de exames "post-mortem" (Marsh et al., 1977), provenientes de seis carcaças de peixes-boi marinhos resgatados pelo Centro Mamíferos Aquáticos (CMA)/IBAMA, decorrentes de captura acidental, sendo machos e fêmeas, oriundas dos Estados do Rio Grande do Norte, Paraíba e Alagoas. Além destas, foram obtidas amostras fecais de cinco peixes-boi marinhos reintroduzidos e 11 amostras provenientes de espécimes nativos, com ocorrência no litoral Norte do Estado da Paraíba.Todo o material biológico coletado foi acondicionado em solução de formalina a $10 \%$, devidamente identificado e encaminhado para a Unidade Executora do CMA/IBAMA, no Estado da Paraíba. Em seguida, foram separados os fragmentos alimentares, com auxílio de placa de petri, agulha $(25 \times 7)$ e $15 \mathrm{~mL}$ de água.

Após a triagem do material, as amostras foram encaminhadas ao Laboratório de Algas Marinhas, da Universidade Federal da Paraíba e submetidas à identificação qualitativa a nível de gênero e/ou espécie, através de aspectos morfo-anatômicos, utilizando estereomicrosccópico óptico (Hurst e Beck, 1988; Wynne, 2005).

\section{Resultados e Discussão}

Entre as estruturas identificadas na dieta dos peixesboi marinhos, foi constatada a presença de algas verdes (Caulerpa prolifera, C. mexicana, C. cupressoides, C. sertularioides e Anadyomene stellata), pardas (Dictyopteris sp., Dictyota sp., Padina gymnospora e Sargassum sp.), ver- 
melhas (Cryptonemia crenulata, Bryothamnion seaforthii, Osmundaria obtusiloba, Hypnea musciformis, Gelidiella acerosa, Gelidium sp., Gracilaria sp., G. domingiensis), fanerógamas (Halodule wrightii, Halophila sp.) e cnidários, sendo observado de dois a dez itens alimentares por amostra, onde o consumo médio por animal em cada amostra foi equivalente a seis itens. Para cada amostra analisada, os diferentes itens identificados foram considerados como uma nova ocorrência, sendo computados 21 taxa ou grupos (13 espécies, 6 gêneros, 1 filo, 1 grupo), entre organismos vegetais e animais (Tabela 1 ).

A variedade de itens alimentares ingeridos entre os sirênios, também foi observada na dieta de dugongos na Austrália (Marsh et al., 1982; Sheppard et al., 2007), peixes-boi marinhos na Flórida (Hartman, 1979; Reich e Worthy, 2006) e peixes-boi amazônicos (Colares e Colares, 2002). De acordo com Colares (1991), os valores nutricionais das plantas aquáticas identificadas na dieta dos peixes-boi amazônicos foram variáveis, de modo que a diversidade destas macrófitas pode proporcionar um melhor aporte às exigências nutricionais requeridas para esta espécie.
Na região Amazônica, a diversidade de macrófitas encontradas durante a seca na dieta dos peixes-boi amazônicos foi apontada como decorrente da menor disponibilidade de plantas, o que diminuiu a disponibilidade de biomassa, já no período da cheia, a maior disponibilidade alimentar proporcionou uma dieta mais seletiva (Colares e Colares, 2002). No que concerne às amostras analisadas neste estudo, em duas ocasiões distintas, o reduzido número de itens alimentares esteve associado aos animais diagnosticados com alterações patológicas.

Lima (1997) relata que o capim agulha (Halodule sp.) é o principal alimento dos peixes-boi marinhos. No entanto, de acordo com a análise realizada neste estudo, além desta fanerógama, outras espécies mostraram-se relevantes na dieta destes animais, na grande maioria submersas, com predominância das algas vermelhas, a exemplo da Cryptonemia crenulata, Gracilaria sp., Hypnea musciformis. Outras espécies vegetais podem apresentar importância significativa na dieta alimentar destes animais, porém fatores fisiológicos relacionados a digestibilidade destes organismos, bem como suas características histológicas podem dificultar em sua identificação.

TABELA 1: Itens alimentares identificados nas amostras fecais e estomacais de peixes-boi marinhos em vida livre.

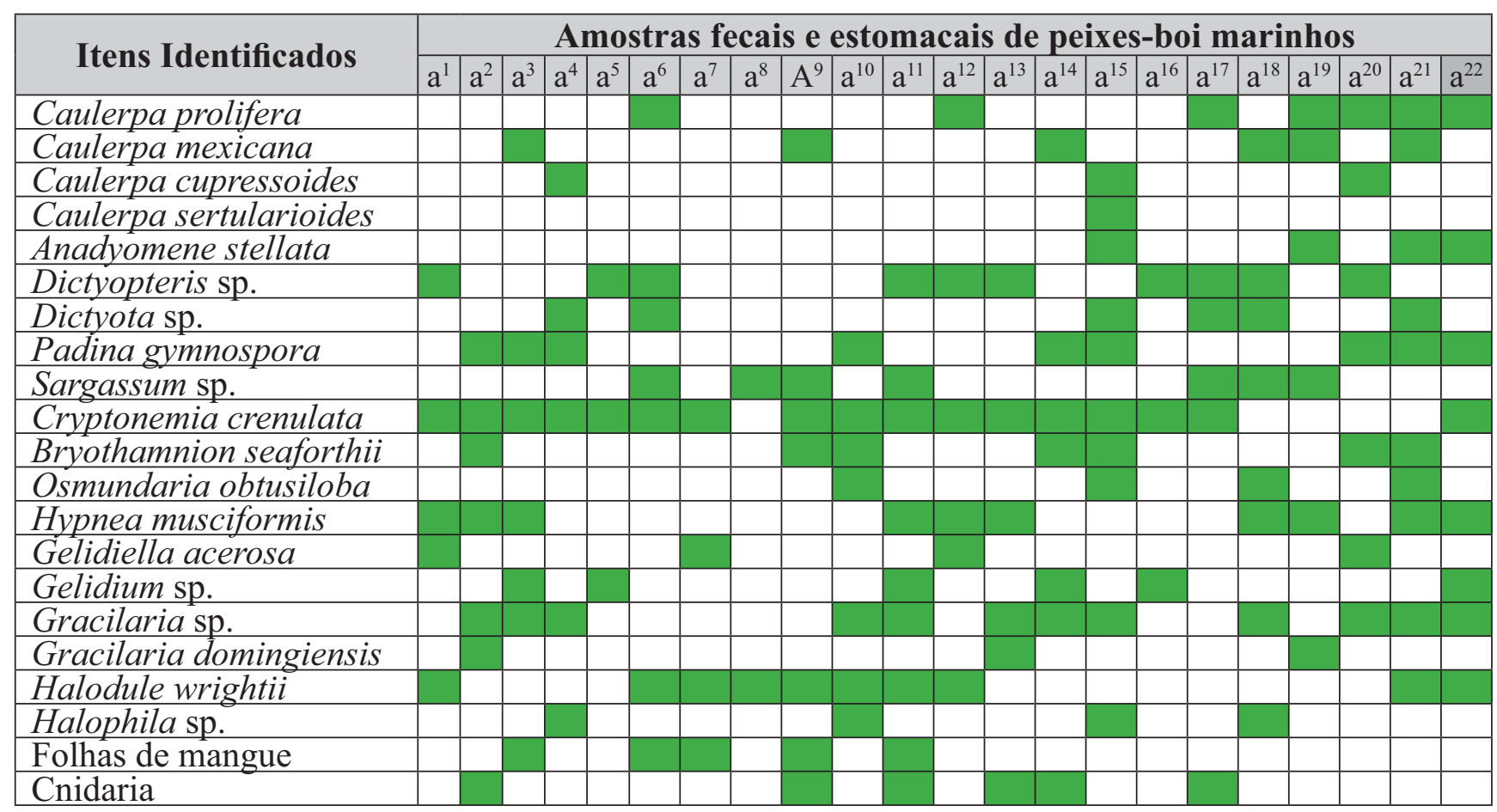

$\mathrm{a}=$ amostra 
Em estudos realizados nas áreas de alimentação dos peixes-boi marinhos no Estado do Rio Grande do Norte, foram coletadas 29 espécies de algas, integrantes da densa cobertura vegetal do local, sendo constatado a maior prevalência das algas vermelhas (Paludo, 1997). A freqüência de ocorrência destas macrófitas coincide com o constatado por Carvalho (1980), ao observar a maior diversidade e abundância destas nos platôs recifais da Paraíba.

Sendo assim, o predomínio de algas vermelhas, encontradas nas amostras analisadas, pode estar associado à preferência alimentar dos peixes-boi marinhos, conforme estudos realizados em cativeiro (Paludo, 1997; Rêgo, 1998), mas, sobretudo, a biomassa existente nas áreas de alimentação, tendo em vista o seu comportamento de herbivoria oportunística (Hartman, 1979). Evidências semelhantes foram relatadas por Marsh et al. (1982) ao relatar que o item de maior freqüência encontrado na dieta de dugongos apresentava-se abundante nas áreas marinhas utilizadas por estes animais.

Além dos vegetais, a identificação dos cnidários reforça as evidências da ingestão involuntária de invertebrados, mediante a presença destes nas macrófitas. Constatações semelhantes foram reportadas na dieta de dugongos na Austrália e peixes-boi marinhos na Flórida e Jamaica, após o consumo acidental de larvas de insetos, anfíbios, moluscos, crustáceos e peixes que permaneciam em meio à vegetação aquática (Powell, 1978; O’Shea et al., 1991; Preen, 1995). Apesar de em alguns casos não haver a intenção da ingestão (Courbis e Worthy, 2003), foi hipotetizado que estes organismos contribuem substancialmente como suplementação de nutrientes (Best, 1981), principalmente de proteínas (Hartman, 1979).

As amostras fecais e conteúdos estomacais coletados foram provenientes dos Estados de Alagoas, Paraíba e Rio Grande do Norte, sendo estes locais uma das principais áreas de ocorrência dos peixes-boi marinhos no litoral Nordeste do Brasil (Lima, 1997; Paludo, 1997). A utilização destes locais por estes animais é favorecida pela presença de bancos de macroalgas (Araújo et al., 2004), fanerógamas marinhas (Lima, 1997) e organismos da meiofauna associado a estes vegetais (Oliveira et al., 2003), pois de acordo com Luna (2001), a disponi- bilidade alimentar influencia positivamente a presença dos peixes-boi marinhos, despertando a necessidade eminente de proteção destes ambientes.

Os diferentes organismos vegetais e animais consumidos pelos peixes-boi marinhos de vida livre reportam para uma alimentação diversificada, de modo que a identificação dos itens constituintes vem a contribuir na adoção da dieta mais apropriada a ser ofertada para espécimes mantidos em cativeiros, tornando oportuno posteriormente a avaliação nutricional destes itens alimentares.

\section{Agradecimentos}

Os autores agradecem a todos os funcionários e estagiários da Base Avançada do Centro Mamíferos Aquáticos/ICMBio no estado da Paraíba, por toda contribuição nas atividades de coleta e triagem das amostras; à FACEPE e ao Programa de Pós-Graduação em Ciência Veterinária da Universidade Federal Rural de Pernambuco.

\section{Referências}

Ames, A. L.; Vleet, E. S. V.; Sackett, W. M. 1996. The of stable carbon isotope analysis for determining the dietary habits of the Florida manatee, Trichechus manatus latirostris. Marine Mammal Science, 12 (4): 555-563.

Araújo, P. G.; Fujii, M. T.; Miranda, G. E. C. 2004. Ecologia de Gracilaria birdiae (Gracilariales, Rhodophyta) da Área de Proteção Ambiental da Barra do Rio Mamanguape, Paraíba-Brasil. Anais da X Reunião da Sociedade Brasileira de Ficologia, Salvador, Bahia, CR-Rom.

Baumgartner, L. L.; Martin, A. C. 1939. Plant histology as an aid in squirrel food habitats studies. Journal Wildlife Management, 3 (3): 266-268.

Best, R. C. 1981. Foods and feeding habits of wild and captive Sirenia. Mammal Review, 11: 3-29.

Burn, D. M. 1986. The digestive strategy and efficiency of the West Indian manatee, Trichechus manatus. Comparative Biochemistry and Physiology, 85 (1): 139-142.

Carvalho, F. A. F. 1980. A flora marinha bêntica do litoral do Estado da Paraíba: perspectivas econômicas. Boletim do Instituto Oceanográfico, 29 (3): 83-85.

Colares, I. G. 1991. Hábitos alimentares do peixe-boi da Amazônia (Trichechus inunguis, Mammalia Sirenia). Dissertação de Mestrado, Universidade Federal do Amazonas, Brasil, 110pp.

Colares, I. G.; Colares, E. P. 2002. Food plants eaten by Amazonian manatees (Trichechus inunguis, Mammalia: Sirenia). Brazilian Ar- 
chives of Biology and Technology, 45 (1): 67-72.

Courbis, S. S.; Worthy, G. A. J. 2003. Opportunistic rather than incidental carnivory by Florida manatee (Trichechus manatus latirostris)? Aquatic Mammals, 29: 104-107.

Hartman, D. S. 1979. Ecology and behavior of the manatee Trichechus manatus in Florida. $5^{\mathrm{a}}$ ed. American Society of Mammalogists, Lawrence, USA, 153pp.

Heinsohn, G. E.; Birch, W. R. 1972. Foods and feeding habits of the Dugong, Dugong dugon (Erxleben) in northern Queensland, Australia. Mammalia, 36: 414-422.

Hicks, G. R. F. 1985. Meiofauna associated with rocky shore algae. In: Moore, P. G. \& Seed, R. (eds). The ecology of rocky coasts. Hodder \& Stoughton Ltda, London, UK, p. 36-56.

Hurst, L. A.; Beck, C. A. 1988. Microhistological characteristics of selected aquatic plants of Florida. With technniques for the study of manatee food habits. Biological Report, 88 (18): 1-145.

Ledder, D. A. 1986. Food habits of the West Indian manatee, Trichechus manatus latirostris, in south Florida. Dissertação de Mestrado, Universidade de Miami, USA, 113pp.

Lima, R. P. 1997. Peixe-boi marinho (Trichechus manatus): Distribuição, status de conservação e aspectos tradicionais ao longo do litoral nordeste do Brasil. Dissertação de Mestrado, Universidade Federal de Pernambuco, Brasil, 86pp.

Luna, F. O. 2001. Distribuição, status de conservação e aspectos tradicionais do peixe-boi marinho (Trichechus manatus manatus) no litoral norte do Brasil. Dissertação de Mestrado, Universidade Federal de Pernambuco, Brasil, 122pp.

Matos, C. H. C.; Oliveira, C. R. F.; Lins, P. A. M. 1998. Dados preliminares sobre as feofíceas da praia de São José da Coroa Grande, litoral sul de Pernambuco. Anais do XLIX Congresso Nacional de Botânica, Salvador, Brasil, p.151.

Marsh, H.; Channels, P. W.; Heinsohn, G. E.; Morrisey, J. 1982. Analysis of stomach contents of Dugongs from Queensland. Australian Wildlife Research, 9: 55-67.

Marsh, H.; Heinsohn, G. E.; Spain, A. V. 1977. The stomach and duodenal divertícula of the dugong, Dugong dugon (Muller). In: Harrison, R. J (ed). Comparative Anatomy of Marine Mammals. Academic Press, New York, USA, p.271-295.
Oliveira, C. R. F.; Matos, C. H. C.; Rocha, C. M. C. 2003. Microgastrópodes Caecidae associados às macroalgas Padina gymnospora (Kuetzing) Sonder e Hypnea musciformis (Wulfen) Lamouroux) na praia de Candeias (Jaboatão dos Guararapes, PE). Revista Brasileira de Zoociências, 5 (2): 213-223.

O’Shea, T. J.; Rathbun, G. B.; Bonde, R. K.; Buergelt, C. D.; Odell, D. K. 1991. An epizootic of Florida manatees associated with a dinoflagellate bloom. Marine Mammal Science, 7 (2): 165-179.

Paludo, D. 1997. Estudos sobre a ecologia e a conservação do peixe-boi marinho Trichechus manatus manatus no Nordeste do Brasil. Dissertação de Mestrado, Universidade Federal da Paraíba, Brasil, 94pp.

Paludo, D.; Langguth, A. 2002. Use of space and temporal distribution of Trichechus manatus manatus Linnaeus in the region of Sagi, Rio Grande do Norte State, Brazil (Sirenia, Trichechidae). Revista Brasileira de Zoologia, 19 (1): 205-215.

Powell, J. A. J. 1978. Evidence of carnivory in manatees (Trichechus manatus). Journal of Mammal, 59 (2): 442.

Preen, A. 1995. Diet of dugongs: are they omnivores? Journal of Mammalogy, 76 (1): 163-172.

Rêgo, A. P. F. 1998. Estudo da alimentação do peixe-boi (Trichechus manatus manatus) em semi-cativeiro no estuário do Rio Mamanguape, PB. Monografia, Universidade Federal da Paraíba, Brasil, 51pp.

Reich, K. J.; Worthy, G. A. J. 2006. An isotopic assessment of the feeding habits of free-ranging manatees. Marine Ecology Progress Series, 322: 303-309.

Sheppard, J. K.; Lawler, I. R.; Marsh, H. 2007. Seagrass as pasture for seacows: Landscape-level dugong habitat evaluation. Estuarine, Coastal and Shelf Science, 71: 117-132.

Wake, J. A. 1975. A study of the habitat requeriments and feeding biology of the dugong, Dugong dugon (Muller). Tese de Doutorado, James Cook University, Austrália, 133pp.

Wynne, M. J. 2005. A checklist of benthic marine algae of the tropical and subtropical western Atlantic: second revision. Science Publishers, Stuttgart, Germany, 158pp. 